\title{
Efeitos da Natação e do Treinamento Resistido na Densidade Mineral Óssea de Mulheres Idosas
}

\section{Effects of Swimming and Resistance Training on Bone Mineral Density of Older Women}

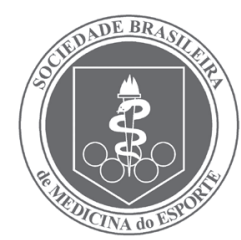

Artigo Original

\author{
Carlos Kemper \\ Ricardo Jacó de Oliveira \\ Martim Bottaro \\ Ricardo Moreno \\ Lídia Mara Aguiar Bezerra \\ Marcelo Guido \\ Nancí Maria de França
}

Universidade Católica de Brasília - Águas Claras, Distrito Federal. Brasil.

Endereço para correspondência: Ricardo Jacó de Oliveira QS. 07 Lote 01, Bloco G Sala 119 - Águas Claras - CEP: 71966-700, Taguatinga - DF

Email: rjaco@ucb.br

Submetido em 16/05/2006 Versão final recebida em 25/10/2006 Aceito em 21/11/2006

\begin{abstract}
RESUMO
Exercícios com impacto como caminhada, saltos, corridas e exercícios resistidos são muito utilizados para prevenção da perda óssea em idosas. No entanto, poucos são os estudos que relatam os efeitos da natação na manutenção da massa óssea em mulheres idosas. Portanto, o objetivo deste estudo foi comparar os efeitos da natação com o treinamento resistido na densidade mineral óssea (DMO) de mulheres idosas. Vinte e três mulheres com idade média de 63,9 $\pm 6,49$ anos foram divididas em dois grupos: 1 ) grupo natação (NAT, $n=13$, que) treinou em intensidade entre 60 e 90\% da freqüência cardíaca de reserva; 2$)$ grupo treinamento resistido (TR, $n=10)$, que treinou os principais grupamentos musculares com três séries a 80\% de 1RM. Os dois grupos praticaram três vezes por semana com uma hora de duração para cada sessão, durante seis meses. A DMO do colo do fêmur e da coluna lombar (L2-L3-L4) foi mensurada através de DXA antes (T0) e após seis meses de treino (T6). Os resultados mostraram que as médias para a DMO lombar em T0 $\left(0,9250 \pm 0,1506 \mathrm{~g} / \mathrm{cm}^{2}\right)$ e T6 $\left(0,9303 \pm 0,1269 \mathrm{~g} / \mathrm{cm}^{2}\right)$ para o NAT e em TO $\left(0,9739 \pm 0,1249 \mathrm{~g} / \mathrm{cm}^{2}\right)$ e T6 $\left(0,9737 \pm 0,1317 \mathrm{~g} / \mathrm{cm}^{2}\right)$ para o TR não foram diferentes quando comparadas intra ou intergrupos. De modo similar, não houve diferenças entre a DMO do colo do fêmur em TO $(0,7784 \pm$ $\left.0,1523 \mathrm{~g} / \mathrm{cm}^{2}\right)$ eT6 $\left(0,7905 \pm 0,1610 \mathrm{~g} / \mathrm{cm}^{2}\right)$ para o NAT e T0 $\left(0,7546 \pm 0,1360 \mathrm{~g} / \mathrm{cm}^{2}\right)$ eT6 $\left(0,7522 \pm 0,1421 \mathrm{~g} / \mathrm{cm}^{2}\right)$ para oTR. Os resultados deste estudo não demonstraram diferenças na DMO entre NAT eTR após seis meses de treino; e que tanto TR quanto NAT não produzem aumentos significativos na DMO de mulheres idosas nesse período.
\end{abstract}

Palavras-chave: pós-menopausa, osteoporose, exercício, massa óssea, DXA.

\begin{abstract}
Impact exercises like walking, jumping, running and resistance exercises are largely used to prevent bone loss in older women; however, few studies report the benefits of swimming to bone tissue. Therefore, the purpose of this study was to compare the effects of swimming with resistance training on bone mineral density (BMD) of older women. Twenty-three women with mean age of $63.9 \pm 6.49$ years were divided in two groups: 1) Swimming group (SWM, $n=13$ ) trained at intensity between 60 and $90 \%$ of heart hate reserve; 2) Resistance training group ( $R T, n=10$ ) trained the main muscular groups with three sets at $80 \%$ of $1 R M$. Both groups trained 3 days/week, one hour per session, during six months. Femoral neck and lumbar spine (L2-L3-L4) BMD were assessed by DXA before (T0) and after six months (T6) of training. Results showed that mean BMD at lumbar spine at T0 $\left(0.9250 \pm 0.1506 \mathrm{~g} / \mathrm{cm}^{2}\right)$ and T6 $\left(0.9303 \pm 0.1269 \mathrm{~g} / \mathrm{cm}^{2}\right)$ for SWM, and at T0 $\left(0.9739 \pm 0.1249 \mathrm{~g} / \mathrm{cm}^{2}\right)$ and T6 $\left(0.9737 \pm 0.1317 \mathrm{~g} / \mathrm{cm}^{2}\right)$ for RT were not different when inter and intracompared. Similarly, there were no differences in femoral neck BMD at T0 $\left(0.7784 \pm 0.1523 \mathrm{~g} / \mathrm{cm}^{2}\right)$ and T6 $\left(0.7905 \pm 0.1610 \mathrm{~g} / \mathrm{cm}^{2}\right)$ for SWM, or T0 $\left(0.7546 \pm 0.1360 \mathrm{~g} / \mathrm{cm}^{2}\right)$ and T6 $\left(0.7522 \pm 0.1421 \mathrm{~g} / \mathrm{cm}^{2}\right)$ for RT. The results of this study showed no difference in BMD between RT and SWM after six months of training. Additionally, neither RT nor SWN produced significant increases in BMD of older women.
\end{abstract}

Keywords: post-menopause, osteoporosis, exercise, bone mass, DXA.

\section{INTRODUÇÃO}

O avanço da idade está associado a desequilíbrio entre a reabsorção e formação óssea, levando a progressivo declínio na densidade mineral óssea (DMO). Em conjunto com a deterioração das trabéculas dos ossos, esse processo tem como conseqüência fragilidade óssea e aumento do risco de fraturas em pessoas idosas ${ }^{(1)}$. As pessoas mais acometidas são as mulheres, pois na condição de menopausa ou pós-menopausa esse declínio é mais acelerado e uma das formas para prevenção, manutenção e aquisição de massa óssea é o exercício físico ${ }^{(2)}$. O exercício físico tem importante papel na prevenção dessas alterações, evidenciando que maiores valores de DMO estão relacionados com estilos de vida mais ativos fisicamente ${ }^{(3-8)}$ e menor incidência de fraturas, diminuindo assim a mortalidade e morbidade ${ }^{(1,9)}$.

A carga proveniente do exercício físico pode ser causada por forças gravitacionais, tais como caminhada, corrida, dança ou por contrações musculares como, por exemplo, a natação e a hidroginástica. Nesse sentido, atividades que produzem maiores estímulos ao tecido ósseo

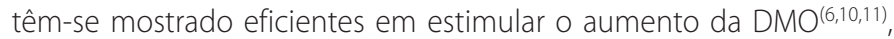
como o treinamento resistido, o qual consiste em uma atividade voltada para o desenvolvimento das funções musculares através da aplicação de sobrecargas, podendo esta ser imposta através de pesos livres, máquinas específicas, elásticos ou a própria massa corporal. 
Por outro lado, atividades sem impacto como a natação têm trazido efeitos conflitantes. Em um estudo recente, Nikander et al. ${ }^{(7)}$ compararam atletas e não atletas. Os atletas eram praticantes de esportes de impacto (esqui, levantamento de peso, vôlei, futebol, corrida orientada) e praticantes de esportes sem impacto (natação e ciclismo) Os resultados demonstraram que todos os atletas dos esportes com impacto apresentaram DMO do colo do fêmur maior em comparação com os não atletas. Adicionalmente, foi observado que os atletas da natação e ciclismo tinham a DMO menor comparada com a dos de esportes de impacto, porém, não diferiram significativamente em relação aos não atletas. achados similares aos de outros estudos ${ }^{(13-15)}$. Em contraste, Tsukahara et al. (16) sugerem que a participação em esportes aquáticos é um fator importante na prevenção da perda de material ósseo. Isso supõe que, mesmo não havendo a força gravitacional como no treinamento resistido, somente o trabalho de contração muscular poderia estar contribuindo para alterações da massa óssea. Esses resultados conflitantes podem ser devidos a fatores não controlados, como diferenças na característica do treinamento tais como: intensidade, duração, freqüência e da seleção de indivíduos com um valor de DMO específico. Nesse sentido, esse estudo teve o propósito de comparar o efeito da natação e do treinamento resistido sobre a DMO de mulheres em fase pós-menopausa.

\section{MÉTODOS}

\section{Amostra}

Foram recrutadas 30 mulheres voluntárias que já se encontravam na fase pós-menopausa, não praticantes de atividade física regular por meio de publicidade impressa e distribuída aos idosos que participam do projeto de extensão que atende a população idosa com atividades físicas e recreativas. As participantes foram randomizadas em dois grupos: natação $(n=15)$ e treinamento resistido $(n=15)$. No grupo NAT, duas participantes foram excluídas do estudo, uma devido a referir dores no peito durante as sessões e a outra por faltar a mais de $25 \%$ dos treinos. No grupo TR, cinco participantes abandonaram o estudo: duas por mudança de domicílio, uma por não ter realizado o exame de DXA e duas por não freqüentarem mais de $75 \%$ das sessões de treinamento. Conseqüentemente, os grupos NAT e TR concluíram o estudo com 13 e 10 participantes, respectivamente. O estudo foi aprovado pelo Comitê de Ética da Universidade Católica de Brasília e os participantes assinaram um termo de consentimento livre e esclarecido, o qual continha informações sobre a natureza voluntária da amostra, o sigilo dos dados e o direito de abandonar o estudo a qualquer momento.

\section{Procedimentos}

Todas as medidas de DMO, antropometria e composição corporal foram realizadas no laboratório de imagens e no Laboratório de Estudos em Educação Física e Saúde (LEEFS) da Universidade Católica de Brasília (UCB-DF).

\section{Densidade mineral óssea (DMO)}

A DMO da coluna lombar (L2-L3-L4) e colo do fêmur foi avaliada através do método de absortometria por raios $X$ de dupla energia (DXA) em um densitômetro modelo Lunar DPX-IQ (Lunar Corporation, Madison, WI, EUA). A estimativa de precisão do método (coeficiente de variação) foi descrita em ser 2,0\% para o colo do fêmur e 1,0\% para a coluna lombar, de acordo com Johnson \& Dawson-Hughes ${ }^{(17)}$. A DMO foi expressa em gramas por centímetro ao quadrado $\left(\mathrm{g} / \mathrm{cm}^{2}\right)$. Os sítios foram escolhidos devido a sua relevância clínica e reprodutibilidade.

\section{Antropometria e composição corporal}

A massa corporal foi mensurada utilizando-se uma balança digital (mod 2006pp Toledo, Brasil) com variação de 0,1 kg, após remoção dos sapatos e com os voluntários vestidos de roupas leves. A estatura foi mensurada utilizando-se um estadiômetro com variação de 0,1cm (Cardiomed, Brasil).

A composição corporal foi mensurada através do DXA, utilizando o equipamento da marca Lunar, modelo DPX-IQ (Lunar Corporation, Madison, WI, EUA). Segundo Hansen et al.(18), o método é considerado válido para avaliar a composição corporal de mulheres idosas, podendo ser aplicado para mensurar a massa livre de gordura (MLG) dessa população. Adicionalmente, Wang et al. ${ }^{(19)}$ validaram mensuração de massa muscular através do DXA contra valores obtidos com a tomografia computadorizada e relataram diferença inferior a 5\%.

Para o procedimento, as voluntárias deitavam em decúbito dorsal sobre a mesa do equipamento, sendo em seguida cuidadosamente posicionadas de forma que ficassem totalmente centralizadas em relação às laterais da mesa. As voluntárias se dispuseram com os membros inferiores estendidos, sendo utilizada uma fita de velcro para mantê-los próximos e dar suporte aos pés, de forma que ficassem numa angulação de $45^{\circ} \mathrm{com}$ relação ao plano vertical. Os membros superiores foram dispostos estendidos e posicionados ao longo do corpo, sem que houvesse contato com o tronco. Todas as avaliações foram realizadas pelo mesmo técnico, o qual foi treinado para realização desses exames, possuindo experiência no procedimento. Após análise de toda a área corporal, o DXA possibilita a determinação da densidade mineral óssea e dos tecidos. Os tecidos são ainda fracionados em massa de gordura e massa livre de gordura.

\section{Freqüência cardíaca de reserva (FCres)}

A FCres foi mensurada com a finalidade de prescrição da intensidade dos treinos de natação. A FCres foi calculada como a diferença entre a freqüência cardíaca de repouso e a freqüência cardíaca máxima. A freqüência cardíaca de repouso foi mensurada através de freqüencímetros torácicos (Polar CIC Inc., Port Washington, NY) com os indivíduos sentados em repouso por cinco minutos e a freqüência cardíaca máxima foi estimada pela fórmula "220 - idade".

\section{Teste de uma repetição máxima (1RM)}

A carga referente a 1 RM foi mensurada de acordo com o protocolo proposto por Kraemer \& Fry ${ }^{(20)}$. Para minimizar a ocorrência de ganhos artificialmente altos causados pela aprendizagem motora, um período de duas semanas de adaptação foi utilizado antes dos testes iniciais, conforme proposto por Ryan et al.(21). Durante as semanas de adaptação, os exercícios foram realizados com cargas leves, com o objetivo de familiarizar as participantes com os equipamentos e com a técnica correta de execução dos exercícios. O teste teve como finalidade a prescrição precisa da intensidade do treinamento resistido.

\section{PROTOCOLOS DE TREINAMENTO}

\section{Natação (NAT)}

O treinamento de natação foi dividido em três mesociclos com duração de dois meses cada. 010 mesociclo foi composto por atividades de adaptação de condicionamento físico geral, tanto aeróbio como neuromuscular, aquisições de habilidades aquáticas e noções do meio líquido. $02^{\circ}$ mesociclo foi composto por atividades para melhoria do condicionamento físico aeróbio e neuromuscular, desenvolvimento das técnicas de nado crawl e costa e melhoria das habilidades aquáticas. O $3^{\circ}$ mesociclo foi composto por atividades que promovem melhoria de força muscular, aquisição de velocidade de nado e manutenção do condicionamento geral e neuromuscular. Os exercícios aquáticos foram realizados durante uma hora, três vezes por semana, num período de seis meses, com a intensidade de 60 a 90\% da freqüência cardíaca de reserva. As aulas tiveram progressividade em relação à intensidade; 
iniciaram-se com atividades de média intensidade (60\% da freqüência cardíaca de reserva) até atingirem alta intensidade (90\% da freqüência cardíaca de reserva).

As aulas foram divididas respeitando a seguinte estrutura: os 10 primeiros minutos utilizados para o aquecimento dos indivíduos; 40 minutos da parte principal foram divididos em: 15 minutos de exercícios (ex.: batida de perna, movimentação de braço, trabalho de respiração), 10 minutos de exercícios para melhoria da coordenação do nado, e 15 minutos para a prática do nado contínuo; e nos 10 minutos finais, para a volta à calma, foram realizados jogos de integração e atividades relaxantes. Também foram programadas algumas atividades de confraternização. A atividade foi desenvolvida em uma piscina com profundidade de $1,50 \mathrm{~m}$ e a temperatura da água durante as aulas permaneceu entre $27^{\circ}$ e $29^{\circ} \mathrm{C}$.

\section{Treinamento resistido (TR)}

Após o período de testes e adaptação, foram realizadas três sessões semanais, em dias não consecutivos, durante seis meses. As sessões de exercícios foram compostas por três séries de 10 repetições, com a carga equivalente a $80 \%$ de 1 RM. Para o reajuste preciso das cargas, o teste de 1RM foi aplicado a cada quatro semanas. No início de cada sessão as participantes realizavam 10 minutos de aquecimento, composto por exercícios de alongamento. Os exercícios realizados foram os seguintes: supino reto, puxada, rosca direta, tríceps, extensão de joelhos, panturrilha, leg press, abdução de ombros e abdômen. 0 exercício de abdômen foi incluído no treinamento, embora não tenha sido realizado o teste de 1 RM com esse grupamento muscular. As participantes foram instruídas a respirar confortavelmente, evitando a manobra de Valsalva.

\section{Análise estatística}

Os dados foram apresentados através de procedimentos de estatística descritiva, em médias e desvios padrão. As diferenças na $\mathrm{DMO}$, massa livre de gordura e no percentual de gordura foram analisadas através da análise de variância de medidas repetidas (ANOVA two ay) com os grupos (TR e NAT) como fatores intersujeitos e o tempo (T0 e T6) como fatores intra sujeitos. O nível de significância foi estabelecido em $p<0,05$.

\section{RESULTADOS}

Os dados descritivos da amostra estão apresentados na tabela 1. Não houve diferenças entre os grupos para os valores iniciais de massa corporal, altura ou idade.

Os resultados da ANOVA não mostraram diferenças intra ou entre grupos para os valores de percentual de gordura, massa livre de gordura e DMO na coluna lombar e do colo do fêmur (tabela 2).
Tabela 1. Médias e desvios padrões das características física da amostra

\begin{tabular}{c|c|c}
\hline Variável & NAT $(n=13)$ & TR $(n=10)$ \\
\hline Idade (anos) & $66,4 \pm 5.3$ & $60,8 \pm 6,8$ \\
\hline Massa corporal $(\mathrm{kg})$ & $60,7 \pm 11,4$ & $63,5 \pm 9,3$ \\
\hline Estatura $(\mathrm{cm})$ & $149,3 \pm 6,9$ & $154,6 \pm 3,8$ \\
\hline Índice de massa corporal $\left(\mathrm{kg} / \mathrm{m}^{2}\right)$ & 27,34 & 26,79 \\
\hline
\end{tabular}

NAT (natação) e TR (treinamento resistido)

\section{DISCUSSÃO}

No presente estudo, nenhum dos grupos apresentou mudanças significativas na DMO após seis meses de treinamento. Uma das possíveis causas para essa ausência de resultados pode ser a duração do estudo. Kerr et al. ${ }^{(22)}$ relataram que, tanto para alcançar resultados significativos quanto para minimizar a perda óssea em mulheres na pós-menopausa, seria necessário exercitar-se com cargas progressivas de treinamento resistido durante pelo menos 12 meses.

Contrário aos nossos achados, Rhodes et al. ${ }^{(23)}$ verificaram que o treinamento resistido realizado em circuito com três séries de oito repetições a 75\% de 1RM promoveu aumentos significativos na DMO de mulheres idosas após um ano. Vincent \& Braith ${ }^{(24)}$ usaram um protocolo similar ao do presente estudo e verificaram que seis meses de treinamento de força a 80\% de 1RM promoveram aumentos na DMO do colo do fêmur em indivíduos idosos; no entanto, o estudo envolveu indivíduos de ambos os sexos. Nossos resultados estão de acordo com os de Liu-Ambrose et al.(25), os quais não encontraram diferenças na DMO de mulheres idosas com baixa massa óssea após seis meses de treinamento resistido. De modo similar, Ryan et al.(21) não verificaram aumentos na DMO após seis meses de treinamento de força progressivo em mulheres idosas. Em um estudo mais longo, Kerr et al.(22) não verificaram alterações na DMO do colo do fêmur e coluna lombar após dois anos de treinamento resistido realizado com três série de oito repetições máximas. No entanto, tendo em vista a tendência de redução da massa óssea em mulheres no período pós-menopausa, a ausência de ganhos da DMO não deve ser vista como indício de ineficiência. Nesse sentido, os resultados de alguns estudos sugerem que o treinamento resistido pode ter um importante papel na prevenção da perda e, não necessariamente, no ganho de $\mathrm{DMO}(16,27,28)$. Como nosso estudo não teve um grupo controle, tal fato não pode ser verificado.

Apesar de grande parte da literatura abordar a prescrição de treinamento resistido para promover alterações favoráveis da $\mathrm{DMO}$, muitos profissionais de saúde indicam atividades aquáticas para os idosos, baseados na suposta segurança dessas atividades. No presente estudo, a DMO não aumentou no grupo NAT após seis meses de treinamento,

Tabela 2. Médias e desvios padrões para o percentual de gordura corporal, massa livre de gordura, DMO da coluna lombar e colo femoral dos grupos NAT e TR antes e após seis meses de treinamento

\begin{tabular}{|c|c|c|c|c|c|c|}
\hline \multirow{2}{*}{ Variáveis } & \multicolumn{3}{|c|}{ NAT $(n=13)$} & \multicolumn{3}{|c|}{$\operatorname{TR}(n=10)$} \\
\hline & TO & $\mathrm{T} 6$ & $\Delta \%$ & TO & $\mathrm{T} 6$ & $\Delta \%$ \\
\hline$\%$ de gord & $40,8 \pm 5,5$ & $39,4 \pm 5,9$ & $-1,4$ & $40,3 \pm 3,5$ & $39,1 \pm 2,9$ & $-1,26$ \\
\hline MLG (kg) & $36,1 \pm 6,3$ & $36,8 \pm 6,4$ & 0,67 & $37,7 \pm 4,3$ & $38,4 \pm 4,3$ & 0,73 \\
\hline DMO lombar ( $\left./ \mathrm{cm}^{2}\right)$ & $0,9250 \pm 0,150$ & $0,9303 \pm 0,126$ & 0,0053 & $0,9739 \pm 0,124$ & $0,9737 \pm 0,131$ & $-0,0002$ \\
\hline DMO colo ( $\left(\mathrm{g} / \mathrm{cm}^{2}\right)$ & $0,7784 \pm 0,152$ & $0,7905 \pm 0,161$ & 0,0121 & $0,7546 \pm 0,136$ & $0,7522 \pm 0,142$ & $-0,0024$ \\
\hline
\end{tabular}

\% de gord = percentual de gordura, MLG = massa livre de gordura, DMO lombar = densidade mineral óssea da coluna lombar, DMO colo = densidade mineral óssea do colo do fêmur, NAT = natação $\mathrm{TR}=$ treinamento resistido, $\mathrm{T} 0=$ tempo inicia, $\mathrm{T} 6=$ tempo final após seis meses 
mas foi observada manutenção dos valores iniciais, sugerindo um efeito de prevenção na perda de massa óssea. Os resultados estão de acordo com o estudo de Tsukahara et al. ${ }^{(16)}$, no qual foi também verificado esse efeito de prevenção após um ano de atividades aquáticas em mulheres em fase pós-menopausa.

As voluntárias deste estudo encontram-se na pós-menopausa, fase em que as mulheres estão com deficiência de estrogênio, hormônio importante para o anabolismo ósseo. O recrutamento das células formadoras, os osteoblastos, depende de forma direta e indireta do estrogênio(33). No caso da deficiência de estrogênio, o sistema ósseo tenta manter ou aumentar a massa óssea através dos estímulos ósseos gerados pelas contrações musculares durante o exercício. É possível que as adaptações propiciadas pelo treinamento resistido demorem mais tempo para ser percebidas.

Por outro lado, Bonaiuti et al. ${ }^{(30)}$ demonstraram que seis meses de intervenção não são suficientes para verificar alterações na massa
Óssea mensurada pelo DXA. Sugerem que seriam necessários pelo menos 12 meses ou mais, uma vez que esse método é uma medida estática e não reflete o metabolismo ósseo. É possível que a utilização de marcadores bioquímicos ósseos para observar as alterações das atividades das células ósseas seria a mais indicada para futuros estudos de curta duração(31,32).

Os resultados deste estudo permitem concluir que não houve diferenças significativas entre o grupo que realizou o treinamento resistido e o grupo que treinou natação na DMO. Sugere-se que estudos futuros comparem os efeitos da natação e do treinamento resistido em amostras maiores, com grupo controle, num período superior a um ano, pois podem fornecer melhores informações sobre a dinâmica das adaptações ao exercício em longo prazo.

Todos os autores declararam não haver qualquer potencial conflito de interesses referente a este artigo.

\section{REFERÊNCIAS BIBLIOGRÁFICAS}

1. Manolagas SC. Birth and death of bone cells: basic regulatory mechanisms and implications for the pathogenesis and treatment of osteoporosis. Endocr Rev. 2000;21(2):115-37.

2. Neto AMP. Soares A, Urbanet AA, Souza ACA, Ferrari AEM, Amaral B, et al. Consenso Brasileiro de Osteoporose. Rev Bras Reumatol. 2002;42(6):343-54.

3. Krall EA, Dawson-Hughes B. Walking is related to bone density and rates of bone loss. Am J Med 1994;96(1):20-6.

4. Kelley GA. Exercise and regional bone mineral density in postmenopausal women: a metaanalytic review of randomized trials. Am J Phys Med Rehabil. 1998;77(1):76-87.

5. Coupland CA, Cliffe SJ, Bassey EJ, Grainge MJ, Hosking DJ, Chilvers CE. Habitual physical activity and bone mineral density in postmenopausal women in England. Int J Epidemiol. 1999;28(2):241-6.

6. Andreoli A, Monteleone M, Van Loan M, Promenzio L, Tarantino U, De Lorenzo A. Effects of different sports on bone density and muscle mass in highly trained athletes. Med Sci Sports Exerc 2001;33(4):507-11.

7. Vuillemin A, Guillemin F, Jouanny P, Denis G, Jeandel C. Differential influence of physical activity on lumbar spine and femoral neck bone mineral density in the elderly population. J Gerontol A Bio Sci Med Sci. 2001;56(6):B248-53

8. Puntila E, Kröger H, Lakka T, Tuppurainen M, Jurvelin J, Honkanen R. Leisure-time physical activity and rate of bone loss among peri- and postmenopausal women: a longitudinal study. Bone. 2001;29(5):442-6.

9. Feskanich D, Willett W, Colditz G. Walking and leisure-time activity and risk of hip fracture in postmenopausal women. JAMA. 2002;288(18):2300-6.

10. Kelley GA, Kelley KS, Tran ZV. Resistance training and bone mineral density in women: a meta-analysis of controlled trials. Am J Phys Med Rehabil. 2001;80(1):65-77.

11. Kelley GA, Kelley KS. Efficacy of resistance exercise on lumbar spine and femoral neck bone mineral density in premenopausal women: a meta-analysis of individual patient data. J Womens Health (Larchmt). 2004;13(3):293-300.

12. Nikander $\mathrm{R}$, Sievänen $\mathrm{H}$, Heinonen $\mathrm{A}$, Kannus $\mathrm{P}$. Femoral neck structure in adult female athletes subjected to different loading modalities. J Bone Miner Res. 2005;20(3):520-8.

13. Orwoll ES, Ferar J, Oviatt SK, McClung MR, Huntington K. The relationship of swimming exercise to bone mass in men and women. Arch Intern Med. 1989;149(10):2197-200.

14. Creighton DL, Morgan AL, Boardley D, Brolinson PG. Weight-bearing exercise and markers of bone turnover in female athletes. J Appl Physiol. 2001;90(2):565-70.

15. Duncan CS, Blimkie CJ, Cowell CT, Burke ST, Briody JN, Howman-Giles R. Bone mineral density in adolescent female athletes: relationship to exercise type and muscle strength. Med Sci Sports Exerc. 2002;34(2):286-94.

16. Tsukahara N, Toda A, Goto J, Ezawa I. Cross-sectional and longitudinal studies on the effect of water exercise in controlling bone loss in Japanese postmenopausal women. J Nutr Sci Vitaminol (Tokyo). 1994;40(1):37-47.

17. Johnson J, Dawson-Hughes B. Precision and stability of dual-energy X-ray absorptiometry measurements. Calcif Tissue Int. 1991;49(3):174-8.
18. Hansen RD, Raja C, Aslani A, Smith RC, Allen BJ. Determination of skeletal muscle and fat-free mass by nuclear and dual-energy $x$-ray absorptiometry methods in men and women aged 51-84. Am J Clin Nutr. 1999;70(2):228-33.

19. Wang ZM, Visser M, Ma R, Baumgartner RN, Kotler D, Gallagher D, et al. Skeletal muscle mass: evaluation of neutron activation and dual-energy X-ray absorptiometry methods. J Appl Physiol. 1996;80(3):824-31.

20. KraemerWJ, Fry AC. Strength testing: Development and evaluation of methodology. In: Physical Assessment of Human Fitness. Maud PJ \& Fosters C, eds. Champaign, IL: Human Kinetics 1995;115-135.

21. Ryan AS, Treuth MS, Hunter GR, Elahi D. Resistive training maintains bone mineral density in postmenopausal women. Calcif Tissue Int. 1998;62(4):295-9.

22. Kerr D, Morton A, Dick I, Prince R. Exercise effects on bone mass in postmenopausal women are site-specific and load-dependent. J Bone Miner Res. 1996;11(2):218-25.

23. Rhodes EC, Martin AD, Taunton JE, Donnelly M, Warren J, Elliot J. Effects of one year of resistance training on the relation between muscular strength and bone density in elderly women. $\mathrm{Br} J$ Sports Med. 2000;34(1):18-22.

24. Vincent KR, Braith RW. Resistance exercise and bone turnover in elderly men and women. Med Sc Sports Exerc. 2002;34(1):17-23.

25. Liu-Ambrose TY, Khan KM, Eng JJ, Heinonen A, McKay HA. Both resistance and agility training increase cortical bone density in 75- to 85 -year-old women with low bone mass: a 6-month randomized controlled trial. J Clin Densitom. 2004;7(4):390-8.

26. Ryan AS, Ivey FM, Hurlbut DE, Martel GF, Lemmer JT, Sorkin JD, et al. Regional bone mineral density after resistive training in young and older men and women. Scand J Med Sci Sports. 2004;14(1):16-23.

27. Humphries B, Newton RU, Bronks R, Marshall S, McBride J, Triplett-McBride T, et al. Effect of exercise intensity on bone density, strength, and calcium turnover in older women. Med Sci Sports Exerc. 2000;32(6):1043-50.

28. Stengel SV, Kemmler W, Pintag R, Beeskow C, Weineck J, Lauber D, et al. Power training is more effective than strength training for maintaining bone mineral density in postmenopausal women. J Appl Physiol. 2005l;99(1):181-8.

29. Nevill A, Holder R, Stewart A. Do sporting activities convey benefits to bone mass throughout the skeleton? J Sports Sci. 2004;22(7):645-50.

30. Bonaiuti D, Shea B, lovine R, Negrini S, Robinson V, Kemper HC. Exercise for preventing and treating osteoporosis in postmenopausal women. Evid Based Nurs. 2003;6(2):50-1.

31. Junqueira PAA, Fonseca AM, Ariê WMY. Osteoporose. Revista de Ginecologia \& Obstetrícia. 2001;12(3):142-56.

32. Chailurkit LO, Ongphiphadhanakul B, Piaseu N, Saetung S, Rajatanavin R. Biochemical markers of bone turnover and response of bone mineral density to intervention in early postmenopausal women: an experience in a clinical laboratory. Clin Chem. 2001;47(6):1083-8.

33. Lanyon L, Armstrong V, Ong D, Zaman G, Price J. Is estrogen receptor alpha key to controlling bones' resistance to fracture? J Endocrinol. 2004;182(2):183-91. 